\title{
Detection of hemoglobin using hybrid molecularly imprinted polymers/carbon quantum dots-based nanobiosensor prepared from surfactant-free Pickering emulsion
}

\author{
Zhou, Tongchang; Ashley, Jon; Feng, Xiaotong; Sun, Yi
}

Published in:

Talanta

Link to article, DOI:

10.1016/j.talanta.2018.08.030

Publication date:

2018

Document Version

Peer reviewed version

Link back to DTU Orbit

Citation (APA):

Zhou, T., Ashley, J., Feng, X., \& Sun, Y. (2018). Detection of hemoglobin using hybrid molecularly imprinted polymers/carbon quantum dots-based nanobiosensor prepared from surfactant-free Pickering emulsion. Talanta, 190, 443-449. https://doi.org/10.1016/j.talanta.2018.08.030

\section{General rights}

Copyright and moral rights for the publications made accessible in the public portal are retained by the authors and/or other copyright owners and it is a condition of accessing publications that users recognise and abide by the legal requirements associated with these rights.

- Users may download and print one copy of any publication from the public portal for the purpose of private study or research.

- You may not further distribute the material or use it for any profit-making activity or commercial gain

- You may freely distribute the URL identifying the publication in the public portal 


\title{
Detection of hemoglobin using hybrid molecularly imprinted polymers/carbon quantum dots-based nanobiosensor prepared from surfactant-free Pickering emulsion
}

\author{
Tongchang Zhou; Jon Ashley; Xiaotong Feng; Yi Sun* \\ Department of Micro- and Nanotechnology, Technical University of Denmark, Ørsteds Plads, DK- \\ 2800 Kgs, Lyngby, Denmark \\ Corresponding Author*E-mail: Sun.Yi@nanotech.dtu.dk.
}

\begin{abstract}
A simple fluorescent nanobiosensor based on molecularly imprinted polymers (MIPs) and carbon quantum dots (CQDs) was developed for hemoglobin $(\mathrm{Hb})$ detection. The nanocomposites were synthesized by a novel one-pot surfactant-free Pickering emulsion method, in which imprinted cavities complementary to $\mathrm{Hb}$ were formed at the surface of the particles for target recognition, while CQDs were incorporated in the core as the fluorescence probe. We innovatively used the $\mathrm{Hb}$ template as emulsifier to help stabilize the emulsion droplets. The method eliminated the need of surfactant, which greatly simplified Pickering emulsion synthesis procedures, and significantly enhanced the fidelity of molecular imprinting. Moreover, the method provided an easy way to integrate fluorescent probes with MIPs in a single step. The nanobiosensor was utilized for determination of $\mathrm{Hb}$ via fluorescence quenching, and high selectivity and sensitivity were achieved. Under the optimized conditions, a linear range of 0.77-7.7 nM and a detection limit of $0.77 \mathrm{nM}$ were obtained. The resulting nanocomposites were also successfully applied to detect $\mathrm{Hb}$ in the serum samples, which showed good recoveries ranging from $86.8 \%$ to $93.9 \%$.
\end{abstract}

\section{Keywords}

Nanobiosensor, surfactant-free Pickering emulsion, MIPs, carbon quantum dots

\section{Introduction}

Hemoglobin $(\mathrm{Hb})$ is a major component in red blood cells and plays an important role for reversible oxygen carrying and storage. It is important to monitor $\mathrm{Hb}$ as numerous diseases are associated with $\mathrm{Hb}$, such as thalassemia, sickle cell disease and emphysema [1,2]. To get precise measurements of $\mathrm{Hb}$, fluorimetry, chemiluminescence and electrochemical methods have been reported [3-5]. However, a number of drawbacks limit their applications, including the fact that 
they require expensive materials like antibodies as receptors, or require complicated sample preparation procedures.

Recently, much attention has been paid to the development of biosensors based on molecularly imprinted polymers (MIPs) [6-8]. MIPs have been regarded as the most potential artificial recognition materials owing to low cost and high robustness. A few $\mathrm{Hb}$ imprinted polymers have been reported, including hydrogel MIPs, magnetic MIPs etc $[9,10]$. However, not much work has been done to employ Hb MIPs for biosensing applications. One of the primary technical hurdles is the lack of simplistic methodologies to synthesize stable Hb MIPs . For protein templates such as $\mathrm{Hb}$, MIPs are normally prepared with a low amount of cross-linker due to the large molecular size, which lead to low stability and reproducibility. To improve the mechanical strength of MIPs, a hard core is required. Pickering emulsion has been successfully used to prepare MIPs with high mechanical strength, which has been proven to be a good method for $\mathrm{Hb}$ imprinting [11]. The resultant MIPs could selectively extract $\mathrm{Hb}$ from mixed proteins. However, the MIPs were large in size $(20 \mu \mathrm{m})$, the polymerization step could easily cause protein denaturation due to the high reaction temperature $\left(60^{\circ} \mathrm{C}\right)$, and the use of surfactant or silica nanoparticles as droplet stabilizer and subsequent HF etching made the synthesis process both complicated and dangerous. Hence, it is necessary to have a simple Pickering emulsion method that can synthesize sub-micrometer sized particles at room temperature.

Another difficulty with MIP-based biosensors is that since MIPs contain no inherent signaling element, they have to be interfaced with transducers to convert the binding events into measurable signals [4], which is not always trivial. A promising method to address this challenge is to combine MIPs with quantum dots (QDs). QDs are fluorescent semiconductor nanocrystals with significant advantages, such as high luminescence, stability against photo bleaching, and narrow emission bands, which make them good candidates for preparing highly sensitive fluorescent sensors. Intense interests have focused on CdTe QDs-based MIPs firstly [5,12]. However, obvious biological toxicity of QDs prepared from heavy metal has been demonstrated [13]. Recently, carbon quantum dots (CQDs) have attracted a lot of interest due to excellent water solubility, good biocompatibility, low toxicity and excellent optical and chemical stabilities. Besides, the band gap and electronic density of CQDs can be well tuned to improve the quantum yield by means of chemical doping. Among them N-doping is the most popular and simple approach to improve the 
properties of CQDs. It has been shown that the interactions between Hb and CQD can lead to fluorescence quenching [14], whereas CQDs alone has no selectivity. To our best knowledge, there has been no work to demonstrate hybrid MIP/CQD for Hb detection.

In this work, we developed for the first time, a nanobiosensor for Hb detection by embedding CQDs in MIPs using a new one-pot surfactant-free Pickering emulsion method (Scheme 1). We innovatively used $\mathrm{Hb}$ itself as emulsifier to help with the stability of the emulsion, since $\mathrm{Hb}$ has both hydrophobic and hydrophilic properties. With the help of probe sonication, we managed to decrease the size of the emulsified droplets to sub-micrometer scale. The CQDs initially dissolved in the water phase were encapsulated in the droplets during this process. Polymerization was then carried out at room temperature by using benzoyl peroxide (BPO) and N,N-Dimethylanaline (DMA) as the initiators [15]. After washing, imprinted cavities complementary to $\mathrm{Hb}$ in size, shape and function groups were formed on the surface. The technique eliminated the need of surfactants or silica nanoparticles, which greatly simplified Pickering emulsion synthesis procedures, and significantly enhanced the fidelity of molecular imprinting. Moreover, the method provided a simple way to combine MIPs with fluorescent probes. The electron transfer between $\mathrm{Hb}$ and CQDs could lead to fluorescence quenching. The MIP/CQD-based nanobiosensor was applied to selectively detect $\mathrm{Hb}$. A linear range of $0.77-7.7 \mathrm{nM}$ and a detection limit of $0.77 \mathrm{nM}$ were obtained. In addition, the nanobiosensor was successfully employed for Hb detection in serum samples and recoveries ranging from $86.8 \%$ to $93.9 \%$ were achieved.

Scheme 1. Schematic illustration of preparation of MIPs/CQD-based nanobiosensors by one-pot surfactant-free Pickering emulsion.

\section{Method}

\subsection{Materials}

Methacrylic acid (MAA, 98.5\%), sodium dodecyl sulphate (SDS, 98\%) and Triton X-100 (99.5\%) were obtained from Merck (Darmstadt, Germany). Benzoyl peroxide (BPO, 97\%), N,N-dimethylaniline (DMA, 98\%), acrylamide (AAm, 98\%), ethylene glycol dimethacrylate (EGDMA, 98\%), hemoglobin (Hb, MW 65 kDa, pI 6.8-7.0), bovine serum albumin (BSA, MW 69 kDa, pI 4.9) and Trypsin from porcine pancreas (TRP, MW 23.8 kDa, pI 10.2-10.8 ) were purchased from Sigma-Aldrich. Other solvents and inorganic salts were of analytical reagent grade 
and were used without further purification.

\subsection{Synthesis of N-doped CQDs}

$2.8 \mathrm{~g}$ of citric acid and $2.8 \mathrm{~g}$ lysine were dissolved in $25 \mathrm{~mL}$ of water. The resultant solution was then transferred to an autoclave reactor and heated to $200{ }^{\circ} \mathrm{C}$ for 5 hours. The resultant yellow solution was then washed in dichloromethane (DCM) for 3 times to remove the starting materials. Retaining the aqueous layer, the carbon dots were precipitated using acetone to form white solid and pelleted using a centrifuge at 3500 RCF for 15 minutes. Three additional washes using acetone and centrifugation were performed. The resultant $\mathrm{N}$-doped carbon dot pellet was then re-suspended in water and freeze dried to give a crystalline solid.

\subsection{Preparation of MIP/CQD nanocomposites by surfactant-free Pickering emulsion}

The Hb (10 mg) was dissolved in $3 \mathrm{~mL}$ milliQ water, and CQDs (30 mg) were dispersed in the solution. Then a mixture of AAm (100 mg), MAA (136 $\mu \mathrm{L})$ and $\mathrm{NaOH}(3 \mathrm{M}, 250 \mu \mathrm{L})$ were added. For the oil phase, $7 \mathrm{mg}$ of BPO was dissolved in EGDMA (400 $\mu \mathrm{L})$ and toluene (100 $\mu \mathrm{L})$. The emulsification was carried out using a probe sonicator set (Hielscher, Germany) at $10 \%$ of the energy output for $15 \mathrm{~s}$ (3 s on and $3 \mathrm{~s}$ off) after the two phases were mixed. At last, DMA (35 $\mu \mathrm{L}$ ) was added to initiate the polymerization. The solid beads were collected after 16 h-reaction at room temperature and washed with methanol for 2 times. Further template removal was ensured by washing the polymer beads with water containing 10\% acetic acid and 5\% SDS for 6 times. Finally the polymer beads were washed with water for 6 times before further drying. The procedure for the preparation of the MIPs is depicted in Scheme 1. As a reference polymer, non-imprinted polymer (NIP) beads were prepared in the same way as the MIP beads, except that the $\mathrm{Hb}$ in the water phase was replaced by Triton X-100 (10 mg).

\subsection{Characterization of CQDs and MIP/CQD nanocomposites}

Transmission electron microscopy (TEM) images were taken using a Tecnai T20 G2 (FEI, Oregon USA) transmission electron microscope. All samples were filtered using a $1 \mu \mathrm{m}$ syringe filter, and $2.5 \mu \mathrm{l}$ of each sample was deposited on a copper TEM grid prior to analysis. IR spectra was taken using a Spectrum 100 (PerkinElmer, MA, USA). X-ray photoelectron spectroscopy (XPS) analysis was carried out by Thermo Scientific ${ }^{\mathrm{TM}}$ K-Alpha ${ }^{\mathrm{TM}}$ X-ray Photoelectron Spectrometer (XPS) System with an Al K-Alpha (1486 ev) x-ray source.

2.5. Optimization of the experimental parameters 
To investigate the kinetics of $\mathrm{Hb}$ quenching, MIP/CQD nanocomposite solution (1 mg mL ${ }^{-1}$, $150 \mu \mathrm{L}$ ) were mixed with $\mathrm{Hb}$ buffer solution $(15.4 \mathrm{nM}, 150 \mu \mathrm{L}, \mathrm{pH}$ 6.2). At different time intervals, the fluorescence spectra were measured with an excitation wavelength of $330 \mathrm{~nm}$ and an emission wavelength of $380 \mathrm{~nm}$, using a Spark ${ }^{\circledR}$ multimode microplate reader (Tecan, Sweden). The fluorescence quenching effect was indicated by $F o / F$, where $F o$ and $F$ were the fluorescence intensity before and after the addition of $\mathrm{Hb}$, respectively. All measurement were conducted in duplicate.

To investigate the effect of $\mathrm{pH}$ on $\mathrm{Hb}$ quenching, MIP/CQD nanocomposite solution (1 mg $\left.\mathrm{mL}^{-1}, 150 \mu \mathrm{L}\right)$ were mixed with $\mathrm{Hb}$ buffer solution at different $\mathrm{pH}(1.54 \mathrm{nM}, 150 \mu \mathrm{L}, \mathrm{pH}$ 5.5, 6.2, 7.0 and 8.0), respectively. After incubation for 10 mins, the fluorescence spectra were measured as described in section 2.5 .

\subsection{Detection of Hb using MIP/CQD-based nanobiosensor}

Hb with different concentrations (1.54 nM, $3.08 \mathrm{nM}, 7.7 \mathrm{nM}$, and $15.4 \mathrm{nM}, 150 \mu \mathrm{L})$ in PBS buffer (pH 6.2) and MIP/CQD nanocomposite solution (1 mg mL $\mathrm{mL}^{-1}, 150 \mu \mathrm{L}$ ) were sequentially injected into each well of a Nunclon 96-well flat-bottom black microplate. After incubation for 10 mins, the fluorescence spectra were measured as described in section 2.5.

\subsection{Spiked samples}

For detection of $\mathrm{Hb}$ in serum samples, different concentrations of $\mathrm{Hb}(20 \mu \mathrm{L})$ were spiked into the pig serum $(80 \mu \mathrm{L})$ to get with the final concentrations of $0.77 \mathrm{nM}, 1.54 \mathrm{nM}$, and $3.85 \mathrm{nM}$, respectively. The measurement was taken in the same manner as described in section 2.5.

\section{Results and discussion}

\subsection{Synthesis of MIP/CQD-based nanobiosensor by Pickering emulsion}

Protein is a kind of macromolecule with long chains. It can act as surfactant and help keep the emulsion stable, since its nonpolar (hydrophobic) segment is partitioned into the oil phase and its hydrophilic segment is exposed to the aqueous phase. As shown in Scheme 1, during the emulsion formation, most of $\mathrm{Hb}$ stayed on the oil/water interface. As the imprinting process was going on at the interface, imprinted sites were created on or close to the surface. It is a big advantage to avoid the use of surfactants, as they generally interfere with the imprinting procedure and are also difficult to be removed from the resulting MIPs. The combination of benzoyl peroxide (BPO) and $\mathrm{N}, \mathrm{N}$-Dimethylanaline (DMA) was used to initiate the polymerization at room temperature in this 
work. High temperature was circumvented here, so that the structure of $\mathrm{Hb}$ could be kept for long time during imprinting process.

The monomers used here were mixture of MAA and AAm, as MAA showed electrostatic interactions with the template while AAm could form hydrogen bond interactions, which were used successfully for protein imprinting [11,16,17]. By adding CQDs in the water phase, MIP/CQD nanocomposites with recognition cavities on surface were simply prepared by the one-step Picking emulsion polymerization.

\subsection{Characterization of MIP/CQD nanocomposites}

TEM was used to investigate the morphology and size distribution of CQDs, MIP/CQDs and NIP/CQDs. As shown in Fig. 1, the CQDs are spherical in shape and uniform in size, with a diameter of approximately $7 \mathrm{~nm}$. The average diameter of MIP/CQD nanocomposites were 900 $\mathrm{nm}$ with regular appearance and good monodispersion. The sub-micrometer sized MIP/CQD particles provided higher surface area, making it more suitable for biosensing applications.

Fig. 1. TEM of CQDs (a), MIP/CQDs (b) and NIP/CQDs (c).

N-doped carbon dots were characterized using both FT-IR spectroscopy and x-ray photoelectron spectroscopy. The IR spectrum for N-doped carbon dots is shown in Fig. 2a. Peaks at $2939 \mathrm{~cm}^{-1}$ corresponds to the $\mathrm{v}(\mathrm{C}-\mathrm{H})$ while the peaks at $1590 \mathrm{~cm}^{-1}$ and $1413 \mathrm{~cm}^{-1}$ correspond to $\mathrm{v}(\mathrm{N}-\mathrm{C}=\mathrm{O})$ and $\mathrm{v}(\mathrm{C}-\mathrm{O}-\mathrm{C})$ respectively. These results suggested the presence of nitrogen and epoxide groups on the surface of the carbon dots. The XPS survey scan (Fig. 2b) showed three peaks at $289 \mathrm{eV}$, $401 \mathrm{eV}$ and $529 \mathrm{eV}$ which correspond to C1s, N1s and O1s respectively. Deconvolution of the C1s scan (Fig. 2c) revealed three peaks at $284 \mathrm{eV}, 285 \mathrm{eV}$ and $287 \mathrm{eV}$ which correspond to C-C, C-N and $\mathrm{C}=\mathrm{O}$ respectively. Deconvolution of the N1s scan (Fig. 2d) showed two peaks at $399 \mathrm{eV}$ and $400 \mathrm{eV}$ which corresponds to $\mathrm{C}-\mathrm{N}$ and $\mathrm{N}-\mathrm{H}$ respectively. Overall, these results confirmed the presence of nitrogen and carbon containing species on the surface of the carbon dots. FT-IR of MIP and NIP after polymerization were shown in Fig. 2e. . The peak observed at $2953 \mathrm{~cm}^{-1}$ was due to the presence of methylene group in both MAA and EGDMA. The carbonyl group $\mathrm{C}=\mathrm{O}$ stretching peak was observed in both MIP and NIP at $1721 \mathrm{~cm}^{-1}$ which also originated from MAA and EGDMA. 
Fig. 2. (a) FT-IR of CDs. (b), (c) and (d) XPS survey spectra for CDs. (e) FT-IR of MIP and NIP.

\subsection{Effect of $\mathrm{pH}$ on the fluorescence detection}

For proteins, isoelectric point $(\mathrm{pI})$ is the $\mathrm{pH}$ at which the protein molecule carries no net electrical charge in the statistical mean. Since the surface properties of protein are affected by the $\mathrm{pH}$ of the solution, it plays an important role in the interaction between $\mathrm{Hb}$ and the MIP/CQD nanocomposites. Quenching experiments over the $\mathrm{pH}$ range of 5.5-8.0 were carried out. All the measurements were conducted after 10 min incubation as the binding reached saturation point after 10 min (Fig. 3a). The response time was much shorter compared with other MIP-based sensors [18,19], which proved that the cavities were located on the surface. It can be seen from Fig. $3 \mathrm{~b}$ that the optimum $\mathrm{pH}$ value for the detection was 6.2. The fluorescence quenching efficiency of CQDs to $\mathrm{Hb}$ was remarkably affected by the $\mathrm{pH}$ due to the electrostatic interaction. The $\mathrm{pI}$ value of $\mathrm{Hb}$ is between 6.8 and 7.0. At higher $\mathrm{pH}$, the zeta potential of CQDs was negative whereas the HB was neutral or negative charged, which could cause weak electrostatic interaction.

Fig. 3. (a) Kinetic quenching of MIP to $\mathrm{Hb}$ at concentration of 7.7 nM. (b) Effect of $\mathrm{pH}$ on the quenching of MIP and NIP to $\mathrm{Hb}$ at concentration of $0.77 \mathrm{nM}$.

\subsection{Sensitivity of $\mathrm{Hb}$ detection using MIP/CQD-based nanobiosensor}

Fluorescence detection of $\mathrm{Hb}$ using MIP/CQD-based nanobiosensor was done under the optimal conditions. As shown in Fig. 4 the fluorescence quenching of MIP/CQDs was much larger than NIP/CQDs under the same condition, which were attributed to the specific molecular recognition sites on the surface of MIPs created during the imprinting process. Moreover, an increase of the $\mathrm{Hb}$ concentration resulted in greater quenching. The extent of the fluorescence quenching of MIPs was linearly related to $\mathrm{Hb}$ concentration in the range of 0.77-7.7 $\mathrm{nM}$. The limit of detection towards $\mathrm{Hb}$ was estimated at $0.77 \mathrm{nM}$. This performance was comparable to other reports as shown in Table 1. 
Fig. 4. Fluorescence emission spectra of MIP/CQDs (a) and NIP/CQDs (b) with Hb of different concentration $(0,0.77,1.54,3.85$ and $7.7 \mathrm{nM})$.

Table 1

Comparison with other report results

\subsection{Selectivity of MIP/CQD-based nanobiosensor}

CQDs have been utilized as a fluorescence probe for direct detection of $\mathrm{Hb}$ in the presence or absence of $\mathrm{H}_{2} \mathrm{O}_{2}$ [14]. The presence of $\mathrm{H}_{2} \mathrm{O}_{2}$ can lead to further improvement of the sensitivity of $\mathrm{Hb}$ detection. However, the use of $\mathrm{H}_{2} \mathrm{O}_{2}$ need to be optimized and made the detection more complicated. Moreover, the lack of selectivity is also a problem. The combination of CQDs with MIPs would greatly enhance the selectivity of $\mathrm{Hb}$. This is extremely important as the sample matrices in real world are usually complex and contain a handful of interfering proteins or contaminants. Here fluorescence quenching of MIP/CQD nanocomposites with other proteins was investigated. As shown in Fig. 5, the fluorescence quenching caused by trypsin (TRP) and bovine serum albumin (BSA) were not obvious compared with $\mathrm{Hb}$. Although BSA is similar to $\mathrm{Hb}$ in size, it could not match the imprinted sites in the shape or functional groups.

Fig. 5. Fluorescence of the MIP and NIP mixed with Hb, TRP and BSA (all set at $3.85 \mathrm{nM}$ ), respectively. Measurement were taken after incubation for 10 mins at $\mathrm{pH} 6.2$.

\subsection{Applications to real samples analysis}

Up to now, the main problem in the detection of $\mathrm{Hb}$ is the low concentration and interfering proteins. It is necessary to increase the sensitivity and selectivity. To validate the applicability of the nanobiosensor, it was employed to analyze $\mathrm{Hb}$ in real serum samples. A recovery study was carried out in the serum samples spiked with different amounts of $\mathrm{Hb}$. Good recoveries in the range of 83.7-93.9\% were obtained which was comparable with other reports $[4,20]$ where sample pretreatment and dilution were normally needed. It was worth nothing here that the serum was used directly without dilution or any other pretreatment. It means that nanobiosensor can detect the target protein even in the presence of many kinds of interfering proteins. The results indicated that the proposed method provided good accuracy and low detection limit. Therefore, the fluorescent nanobiosensor is suitable and promising for the rapid analysis of $\mathrm{Hb}$ in complex 
samples.

Table 2

Recovery of $\mathrm{Hb}$ from spiked pig serum samples.

\section{Conclusion}

In this work, a new surfactant-free Pickering emulsion strategy was developed to prepare MIP/CQD-based nanobiosensor for $\mathrm{Hb}$ detection. The use of $\mathrm{Hb}$ itself as emulsifier, the improvement of initiator choice and size control made this method more applicable for protein imprinting. Besides, the MIP/CQD-based nanobiosensor exhibited excellent sensitivity, high selectivity and fast response. The good recoveries from real serum samples further demonstrated that the nanobiosensor holds great promise for Hb determination. The simplicity of this method can help produce the nanobiosensor in a large scale.

\section{Conflicts of interest}

The authors have no conflicts of interest with any individual or agency.

\section{Acknowledgement}

This work was financially supported by the Villum Fonden, Denmark, Project NO. 13153.

\section{Reference}

[1] K.K. Hussain, J.M. Moon, D.S. Park, Y.B. Shim, Electrochemical Detection of Hemoglobin: A Review, Electroanalysis. 29 (2017) 2190-2199.

[2] C.D. Reiter, X. Wang, J.E. Tanus-Santos, N. Hogg, R.O. Cannon, A.N. Schechter, M.T. Gladwin, Cell-free hemoglobin limits nitric oxide bioavailability in sickle-cell disease, Nat. Med. 8 (2002) 1383-1389.

[3] L.Y. Chen, C.C. Huang, W.Y. Chen, H.J. Lin, H.T. Chang, Using photoluminescent gold nanodots to detect hemoglobin in diluted blood samples, Biosens. Bioelectron. 43 (2013) 3844.

[4] B. Sun, X. Ni, Y. Cao, G. Cao, Electrochemical sensor based on magnetic molecularly imprinted nanoparticles modified magnetic electrode for determination of $\mathrm{Hb}$, Biosens. Bioelectron. 91 (2017) 354-358.

[5] Z.S. Traore, S.M. Shah, X. Su, Flow-injection chemiluminescence determination of haemoglobin in the blood, Luminescence. 28 (2013) 56-62.

[6] H. Niu, Y. Yang, H. Zhang, Efficient one-pot synthesis of hydrophilic and fluorescent molecularly imprinted polymer nanoparticles for direct drug quantification in real biological samples, Biosens. Bioelectron. 74 (2015) 440-446. 
[7] A. Pietrzyk-Le, S. Suriyanarayanan, W. Kutner, R. Chitta, M.E. Zandler, F. D’Souza, Molecularly imprinted polymer (MIP) based piezoelectric microgravimetry chemosensor for selective determination of adenine, Biosens. Bioelectron. 25 (2010) 2522-2529.

[8] N. Murase, S. Taniguchi, E. Takano, Y. Kitayama, T. Takeuchi, A molecularly imprinted nanocavity-based fluorescence polarization assay platform for cortisol sensing, J. Mater. Chem. B. 4 (2016) 1770-1777.

[9] X. Shen, T. Zhou, L. Ye, Molecular imprinting of protein in Pickering emulsion, Chem. Commun. 48 (2012) 8198.

[10] M. Zhang, Y. Wang, X. Jia, M. He, M. Xu, S. Yang, C. Zhang, The preparation of magnetic molecularly imprinted nanoparticles for the recognition of bovine hemoglobin, Talanta. 120 (2014) 376-385.

[11] T. Zhou, K. Zhang, T. Kamra, L. Bülow, L. Ye, Preparation of protein imprinted polymer beads by Pickering emulsion polymerization, J. Mater. Chem. B. 3 (2015) 1254-1260.

[12] W. Zhang, X.W. He, Y. Chen, W.Y. Li, Y.K. Zhang, Composite of CdTe quantum dots and molecularly imprinted polymer as a sensing material for cytochrome c, Biosens. Bioelectron. 26 (2011) 2553-2558.

[13] T.T. Fang, X. Li, Q.S. Wang, Z.J. Zhang, P. Liu, C.C. Zhang, Toxicity evaluation of CdTe quantum dots with different size on Escherichia coli, Toxicol. Vitr. 26 (2012) 1233-1239.

[14] A. Barati, M. Shamsipur, H. Abdollahi, Hemoglobin detection using carbon dots as a fluorescence probe, Biosens. Bioelectron. 71 (2015) 470-475.

[15] X. Shen, J. Svensson Bonde, T. Kamra, L. Bülow, J.C. Leo, D. Linke, L. Ye, Bacterial imprinting at pickering emulsion interfaces, Angew. Chemie - Int. Ed. 53 (2014) 10687-10690.

[16] L. Tan, C. Huang, R. Peng, Y. Tang, W. Li, Development of hybrid organic-inorganic surface imprinted Mn-doped ZnS QDs and their application as a sensing material for target proteins, Biosens. Bioelectron. 61 (2014) 506-511.

[17] L. Liu, K. Yang, L. Zhang, Y. Zhang, Protein-imprinted material for the treatment of antibiotic-resistant bacteria, Sci. Bull. 61 (2016) 1890-1891.

[18] Y. Li, Y. Li, M. Hong, Q. Bin, Z. Lin, Z. Lin, Z. Cai, G. Chen, Highly sensitive protein molecularly imprinted electro-chemical sensor based on gold microdendrites electrode and prussian blue mediated amplification, Biosens. Bioelectron. 42 (2013) 612-617.

[19] X. Kan, Z. Xing, A. Zhu, Z. Zhao, G. Xu, C. Li, H. Zhou, Molecularly imprinted polymers based electrochemical sensor for bovine hemoglobin recognition, Sensors Actuators, B Chem. 168 (2012) 395-401.

[20] J.M. Moon, D.M. Kim, M.H. Kim, J.Y. Han, D.K. Jung, Y.B. Shim, A disposable amperometric dual-sensor for the detection of hemoglobin and glycated hemoglobin in a finger prick blood sample, Biosens. Bioelectron. 91 (2017) 128-135. 